\title{
Comparative analysis of cytotoxic $T$ lymphocyte response induced by dendritic cells pulsed with recombinant adeno-associated virus carrying $\alpha$-fetoprotein gene or cancer cell lysate
}

\author{
JUN ZHOU ${ }^{1 *}$, PING MA²*, JUN LI ${ }^{1}$ and WEI SONG ${ }^{1}$ \\ Departments of ${ }^{1}$ Oncology and ${ }^{2}$ Opthalmology, Provincial Hospital Affiliated to Shandong University, \\ Jinan, Shandong 250021, P.R. China
}

Received February 22, 2014; Accepted November 21, 2014

DOI: $10.3892 / \mathrm{mmr} .2014 .3059$

\begin{abstract}
Hepatocellular carcinoma (HCC) is one of the most common and difficult to treat types of cancer worldwide. Antigen-targeted immunotherapy has the potential to be a novel and effective adjuvant for use in HCC. In the present study, recombinant adeno-associated virus carrying the $\alpha$-fetoprotein gene (rAAV/AFP) and cancer cell lysates were used to pulse antigen-presenting dendritic cells (DCs) in order to stimulate a cytotoxic T lymphocyte (CTL) response against HCC. rAAV/AFP-pulsed and cancer cell lysate-pulsed DCs resulted in a mature DC phenotype with high expression of major histocompatibility complex (MHC) class I, MHC class II, CD80, CD83 and CD86 molecules. However, rAAV/AFP-pulsed DCs exhibited superiority over cancer cell lysate-pulsed DCs in terms of stimulating proliferation of $\mathrm{T}$ cells, activating $\mathrm{T}$ cells to secret interferon- $\gamma$ (IFN- $\gamma$ ) and inducing an AFP-specific MHC class I-restricted CTL response. The current data suggest that pulsing of DCs using rAAV/AFP is more effective than the cancer cell lysate-pulsing technique, and that this technique may be used for the development of immunotherapy in AFP-positive HCC.
\end{abstract}

\section{Introduction}

Hepatocellular carcinoma (HCC) is one of the primary causes of cancer-related mortality, with a global incidence of $>500,000$ cases per year (1). The incidence and mortality of this disease have increased amongst all races and in the two genders over the past two decades. HCC has a poor prognosis,

Correspondence to: Dr Wei Song, Department of Oncology, Provincial Hospital Affiliated to Shandong University, 324 Jing Wu Road, Jinan, Shandong 250021, P.R. China

E-mail: songwei1963@hotmail.com

${ }^{*}$ Contributed equally

Key words: adeno-associated virus, dendritic cells, $\alpha$-fetoprotein, hepatocellular carcinoma, immunotherapy and there are few treatment options. Curative surgery and liver transplantation are only available to a small minority of patients with early-stage HCC. The majority of cases are detected at an advanced stage. Other commonly-used therapies are predominantly palliative $(2,3)$.

$\alpha$-fetoprotein (AFP) is an oncofetal antigen. Suppression of AFP synthesis occurs shortly subsequent to birth. However, $50-80 \%$ of adults with HCC exhibit re-expression of AFP during tumor progression $(2,3)$. Human $\mathrm{T}$ cell repertoires are able to recognize AFP-derived peptide epitopes in the context of major histocompatibility complex (MHC) class I molecules and to induce AFP-specific protection $(2,3)$. Therefore, AFP, as a tumor-associated antigen, may be a suitable target for dendritic cell-based cytotoxic T lymphocyte (CTL)-mediated antigen-targeted immunotherapy in HCC.

Pulsing dendritic cells (DCs) with tumor antigen is a versatile approach by which to generate cancer vaccines (4-8). DCs are the most effective type of antigen-presenting cells and are able to stimulate naive $\mathrm{T}$ lymphocytes to initiate a primary immune response (4). DCs are scarce, representing only $0.2 \%$ of total white blood cells. Therefore, a number of protocols in order to procure DCs from peripheral blood mononuclear cells (PBMCs) in vitro have been developed (9). These protocols allow the in vitro manipulation of DCs for clinical and laboratory-based studies (10). Techniques for generating antigen-loaded DCs include pulsing or incubating DCs with cancer cell lysate, utilizing cancer cells undergoing apoptosis, tumor antigen peptide and specific tumor protein, and the delivery of tumor antigen gene into DCs using viral vectors $(5-7,11-15)$.

A phase II clinical trial demonstrated that vaccination with mature autologous DCs pulsed ex vivo with tumor cell line lysate in patients with advanced HCC was safe and well-tolerated, with evidence of antitumor efficacy assessed radiologically and serologically (8). The results from a separate study supported the superiority of lentivirus-AFP-engineered DC vaccine over AFP peptide-pulsed DC vaccine (16). Recombinant adeno-associated virus (rAAV) is one of the safest virus vectors used in gene therapy, as the wild-type virus has never been shown to cause human disease. A further advantage of rAAV is the absence of viral coding sequences, which may diminish the elimination of transduced DCs 
by virus-specific CTL (17-19). In addition, rAAV is able to transduce dividing and nondividing cells, which may allow the transduction of DCs in various states of activation and maturation, from a broad range of sources (19-21). For these reasons above, rAAV rather than a lentivirus was selected as the vector-carrying AFP gene for pulsing DCs in the present study, and was compared with cancer lysate-pulsed DCs.

The HPV-16 E7 antigen gene has been successfully transduced into DCs using rAAV, which resulted in the induction of a CTL response against cervical cancer cells (22). This result indicated that AFP gene delivery into DCs by rAAV may also be an effective approach for priming CTL response against HCC. The aim of the present study was to demonstrate whether rAAV was able to transduce the AFP gene into DCs effectively and prime an AFP-specific MHC-class I-restricted CTL response.

\section{Materials and methods}

Cell lines. The HepG2 and SK-HEP-1 HCC cell lines, and the K562 and HEK293 myelogenous leukemia cell lines were obtained from the American Type Culture Collection (Manassas, VA, USA). The BEL7402 and SMMC7721 HCC cell lines were purchased from the Cancer Research Department of the China Medical Science Institute (Beijing, China). Cells were cultured in complete Dulbecco's modified Eagle's medium or RPMI-1640 medium (Invitrogen Life Technologies, Carlsbad, CA, USA) supplemented with 5 or $10 \%$ fetal bovine serum (Invitrogen Life Technologies). PBMCs obtained from healthy donors were separated using the Ficoll gradient method and cultured in AIM-V medium (Life Technologies, Carlsbad, CA). All blood donors were enrolled by the Provincial Hospital Affiliated to Shandong University (Jinan, China) and provided written informed consent. The study protocol received a priori approval by the Human Research Internal Review Board of the Provincial Hospital affiliated to Shandong University, indicating that the protocol conformed to the ethical guidelines of the 1975 Declaration of Helsinki. The human leukocyte antigen (HLA) haplotype of all donors was HLA-A2.

Construction of the $r A A V$ vector. The wild-type AAV type 2 genome, pSM620, was digested in order to delete the internal AAV sequences from map units 3-97, including the p5 promoter, and a specially designed polylinker was ligated in place, resulting in the rAAV vector plasmid, d13-97 (23). The cytomegalovirus (CMV) enhancer and the SV40 early mRNA polyadenylation signal DNA were derived from the p-enhanced green fluorescent protein (EGFP)-N1 plasmid (Clontech, Mountain View, CA, USA) and inserted into the d13-97 vector. Subsequently, the CMV immediate early promoter was inserted to the d13-97 vector (d13-97/CMVp), which was derived from pEGFP-N1. Human AFP cDNA was amplified using reverse transcription-polymerase chain reaction (RT-PCR). Total mRNA was isolated from the HepG2 cells using an Oligotex mRNA isolation kit (Qiagen, Valencia, CA, USA). Once the first-strand cDNA was generated, PCR amplification of AFP sequence from nucleotides 12 to 1902 was conducted using the following primers: Forward: 5'-CTT CCACCACTGCCAATAAC-3' and reverse: 5'-TTGTCTTCT
CTTCCCCTG-3' (24). The AFP cDNA was then inserted into the d13-97 vectors for $8 \mathrm{~h}$ at $4^{\circ} \mathrm{C}$ in order to generate the rAAV/AFP vector.

Generation of rAAV virus. The $\mathrm{pSH} 3$ plasmid is able to express the AAV type 2 rep and cap genes and the adenovirus type 5 E2A, VA1 and E4 genes, to allow rAAV DNA replication and packaging into viral particles without contaminating the wild-type AAV and adenovirus (25). The rAAV vector was co-lipofected into HEK293 cells with the pSH3 plasmid, and the rAAV was harvested after four days. A one-step column purification technique, which used gravity flow based on its affinity to heparin, without ultracentrifugation, was performed in order to generate the purified rAAV (26). The rAAV was titered by dot blot hybridization as described previously (22).

Generation and pulsing of monocyte-derived DCs. PBMCs were obtained from HLA-A2-positive healthy volunteers, separated by Ficoll density-gradient centrifugation at $400 \mathrm{x} g$ for $20 \mathrm{~min}$ and incubated in six-well culture plates at $37^{\circ} \mathrm{C}$ for $2 \mathrm{~h}$ in AIM-V medium. Following incubation, nonadherent cells were removed, and adherent PBMCs were cultured in AIM-V medium containing 800 units/ml granulocyte macrophage colony-stimulating factor (GM-CSF; R\&D Systems, Minneapolis, MN, USA). Adherent PBMCs were infected with $10^{10} \mathrm{eg} / \mathrm{ml} \mathrm{rAAV/AFP.} \mathrm{Following} \mathrm{incubation} \mathrm{for} 8 \mathrm{~h}$, the medium/virus solution was removed, the cells were washed and fresh AIM-V medium was added to the cultures. Throughout the culture period, $800 \mathrm{IU} / \mathrm{ml}$ GM-CSF was included in the medium. In order to induce the maturation of DCs, $1,000 \mathrm{IU} / \mathrm{ml}$ of human interleukin-4 (IL-4; R\&D Systems) was added at $24 \mathrm{~h}$. This permitted a brief period of monocyte proliferation, which promoted a higher level of rAAV transduction.

Cancer cell lysates were generated by four rapid freeze-thaw cycles of HepG2 cells. Cancer cell lysate-loading of DCs was performed by incubation of DCs with cancer cell lysate in the absence of transfection reagents. In brief, adherent PBMCs were cultured in AIM-V medium containing 800 units/ml GM-CSF and $1000 \mathrm{IU} / \mathrm{ml}$ IL-4. On day three, cancer cell lysates were added $\left(100 \mu \mathrm{g}\right.$ lysate $\left./ 5 \times 10^{5} \mathrm{DCs}\right)$ and incubated with immature DCs. Every two days, the culture medium was replaced with fresh medium containing the cytokines. At day four, $50 \mathrm{IU} / \mathrm{ml}$ of human tumor necrosis factor- $\alpha$ (R\&D Systems) was added into the culture. After six days of culturing, monocyte-derived dendritic cells were harvested from nonadherent and loosely adherent cells. The survival of DCs upon viral infection was tested by trypan blue stain (Sigma-Aldrich, St. Louis, MO, USA).

AFP expression of DCs and cell lines. DCs were harvested at six days post-transduction, and intracellular staining was performed in order to analyze AFP expression in rAAV/AFP-pulsed DCs. In brief, the cells were fixed, permeabilized and incubated with phycoerythrin (PE)-conjugated mouse anti-human AFP monoclonal antibody (1:100; 563002; BD Pharmingen, San Diego, CA, USA). A FACSCalibur flow cytometer (BD Biosciences, San Jose, CA, USA) was used for data acquisitions. For each sample $\geq 10,000$ cells were counted. Lysate-pulsed or untreated DCs were used as a control. AFP expression of the cell lines was tested using the same procedure. 
$\mathbf{A}$

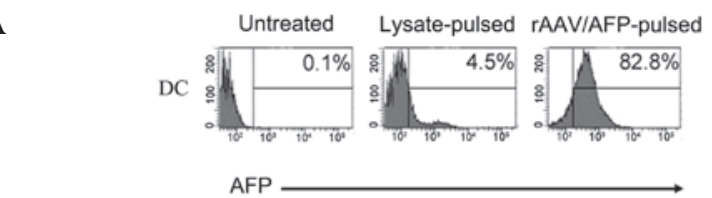

B

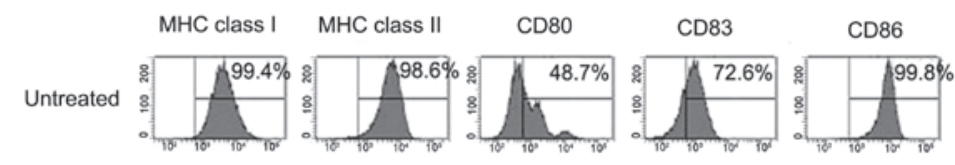

Lysate-pulsed
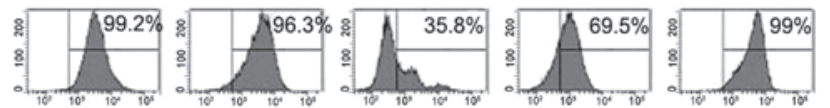

rAAVIAFP-pulsed
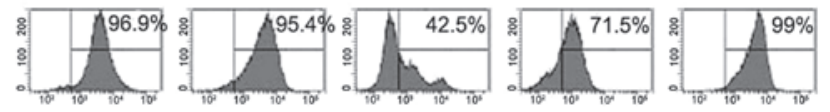

Figure 1. Characterization of DCs pulsed by different techniques. (A) Percentage of AFP-positive DCs. (B) MHC class I, MHC class II, CD80, CD83 and CD86 expression on rAAV/AFP-pulsed, lysate-pulsed or untreated DCs. Percentages of positive cells are shown in the histograms. DCs, dendritic cells; AFP, $\alpha$-fetoprotein; MHC, major histocompatibility complex; rAAV, recombinant adeno-associated virus.

Cell surface marker analysis of DCs and cell lines. For analysis of the phenotype of DCs, the non-adherent and loosely adherent cells were collected by gentle pipetting following six days in culture. Cells were incubated with fluorescin isothiocynate (FITC)-conjugated monoclonal mouse anti-human antibodies directed against MHC class I (HLA-ABC; 1:100; 555552), MHC class II (HLA-DR; 1:100; 555811), CD80 (1:100; 555683), CD83 (1:100; 555910) and CD86 (1:100; 555657), which were all purchased from BD Pharmingen. All samples were then analyzed using a FACSCalibur flow cytometer. HLA-A2 expression of the cell lines were tested using FITC-conjugated mouse anti-human monoclonal antibodies against HLA-A2 (1:100; 551285; BD Pharmingen).

T-cell proliferation assay. $\mathrm{CD} 3+\mathrm{T}$ cells were isolated from PBMCs using a Pan T Cell Isolation kit II (Miltenyi Biotec, Auburn, CA, USA) according to the manufacturer's instructions. The rAAV/AFP-pulsed or lysate-pulsed DCs were co-cultured with $\mathrm{CD} 3+\mathrm{T}$ cells at a ratio of 1:20 in the presence of 20 units $/ \mathrm{ml}$ recombinant human IL-2 and $20 \mathrm{ng} / \mathrm{ml} \mathrm{IL-7}$ (R\&D Systems). The wild type AAV-pulsed and untreated DCs were used as controls. The DC-T cell culture medium was replaced by fresh medium plus the cytokines every two days. On day eight, $\mathrm{T}$ cell proliferation was assessed using the Cell Titer 96 AQueous One Solution Cell Proliferation Assay kit (Promega Corporation, Madison, WI, USA) and ${ }^{3} \mathrm{H}$-thymidine uptake assay. For the Cell Titer 96 AQueous One Solution Cell Proliferation Assay, dye solution was added to each well and incubated for $4 \mathrm{~h}$. A microplate imaging system was used to measure the absorbance of the samples at $490 \mathrm{~nm}$. For ${ }^{3} \mathrm{H}$-thymidine uptake assay, the cultures were pulsed with $1 \mu \mathrm{Ci}{ }^{3} \mathrm{H}$-thymidine (New England Nuclear, Boston, MA, USA) per well for $12 \mathrm{~h} .{ }^{3} \mathrm{H}$-thymidine uptake was counted using an LS 6000SE liquid scintillation counter (Beckman Coulter, Brea, CA, USA).

Cytokine expression level analysis in primed $T$ cells. T cells were harvested eight days after priming. The intracellular staining assay described above was performed to analyze interferon- $\gamma($ IFN- $\gamma$ ) and IL-4 expression using FITC-conjugated mouse anti-human IFN- $\gamma(1: 100 ; 552882)$ and PE-conjugated mouse anti-human IL-4 (1:100; 559333) monoclonal antibodies (BD Pharmingen).

Cytotoxicity assays. After eight days of the DC-T cell culture, $6 \mathrm{~h}$ chromium-51 $\left({ }^{51} \mathrm{Cr}\right)$ release assays were used to analyze the killing activity of the CTL, which had been elicited by the rAAV/AFP-pulsed, lysate-pulsed or untreated DCs against the target cells. The targets included HepG2, BEL7402, SMMC7721, Sk-Hep-1 and natural killer cell (NK)-sensitive K562 tumor cells. The ${ }^{51} \mathrm{Cr}$-labeled target cells were mixed with the CTL (1:20) and incubated for $6 \mathrm{~h}$ at $37^{\circ} \mathrm{C}$ with $5 \% \mathrm{CO}_{2}$. In order to determine the structures on the target cells, the mouse anti-human HLA-A2 monoclonal antibody (1:100; 551230; BD Pharmingen) was used to block cytotoxicity. The ${ }^{51} \mathrm{Cr}$-labeled targets were pre-incubated with mouse anti-human HLA-A2 antibody for $2 \mathrm{~h}$ prior to performance of the ${ }^{51} \mathrm{Cr}$-release assay was. In order to demonstrate the AFP-specific killing activity of the CTL, a series of AFP-negative cells were also tested. K562 cells were used as targets to observe NK activity.

Statistical analysis. All data are expressed as the mean \pm standard deviation and differences between groups were analyzed using the Student's t-test with SPSS 15.0 software (SPSS, Inc., Chicago, IL, USA). $\mathrm{P}<0.05$ was considered to indicate a statistically significant difference.

\section{Results}

Construction and generation of $r A A V$. To construct the rAAV vectors for this study, the CMV immediate early promoters were successfully inserted into the d13-97 vectors. AFP mRNA was isolated from HepG2 cells and amplified by RT-PCR (data not shown). cDNA was successfully cloned into the rAAV vectors, sequenced and determined to be identical to the published sequence $(24,27)$. The viral stocks of rAAV were generated and titered (data not shown). The viral titer was $10^{11} \mathrm{eg} / \mathrm{ml}$. 
A
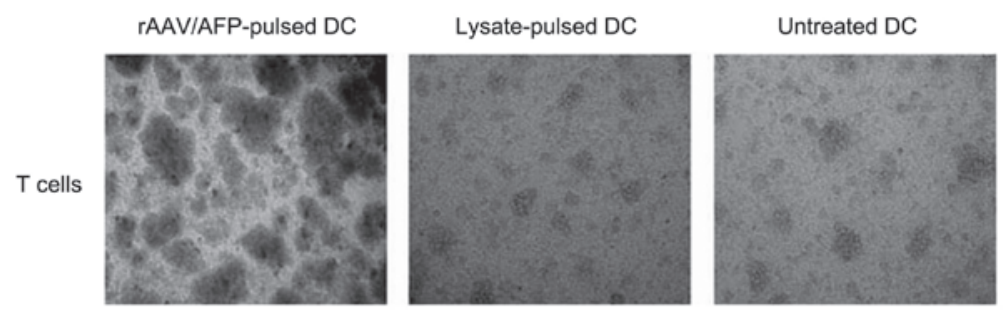

B

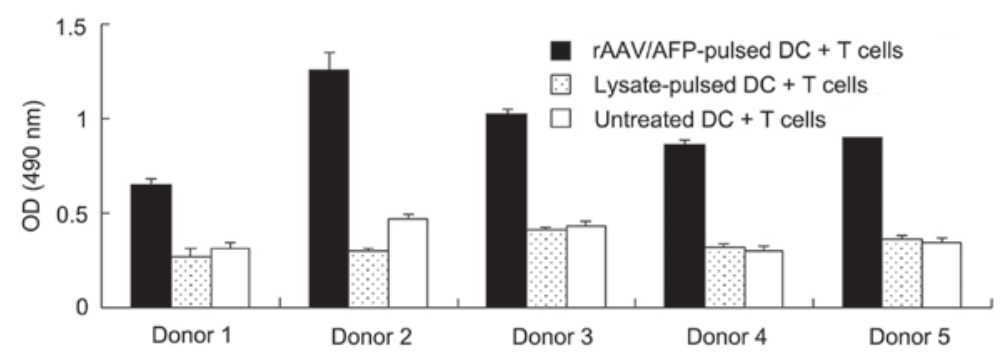

C

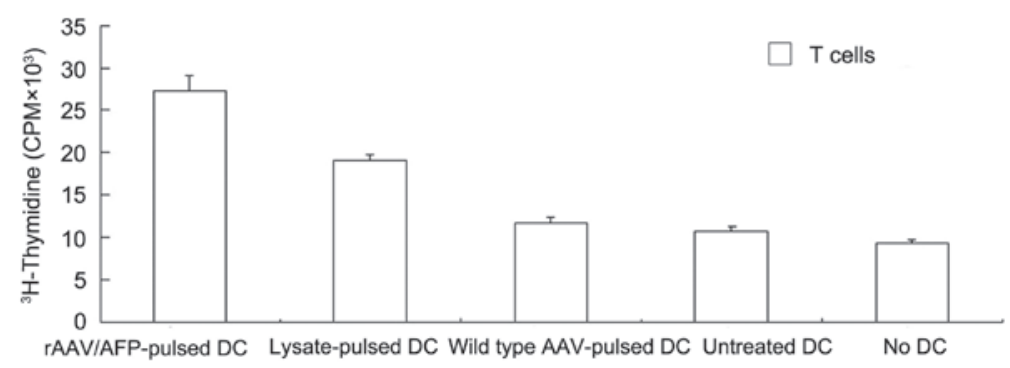

Figure 2. Proliferation of T cells stimulated by DCs pulsed by different techniques. (A) Following the incubation of DCs with T cells, larger cell clusters were observed in rAAV/AFP-pulsed DCs compared with those in the other groups. (B) Proliferation of T cells stimulated by rAAV/AFP-pulsed, lysate-pulsed or untreated DCs. (C) Proliferation of T cells stimulated by rAAV/AFP-pulsed, lysate-pulsed, wild-type AAV-pulsed or untreated DCs. DCs, dendritic cells; AFP, $\alpha$-fetoprotein; rAAV, recombinant adeno-associated virus.

Levels of antigen-positive DCs following rAAV delivery. In order to assess the efficiency of rAAV pulsing of DCs, the level of AFP protein in rAAV/AFP pulsed DCs was analyzed using intracellular staining at six days post-transduction. The results, shown in Fig. 1A, demonstrated that rAAV/AFP infection of DCs results in a high percentage of cells containing intracellular AFP protein (82.8\%).

Characterization of DCs. DCs were generated from healthy volunteers. The phenotypes of DCs were examined by flow cytometric analysis in order to determine whether significant differences in phenotype were discernible among untreated, lysate-pulsed and rAAV/AFP-pulsed DC populations. The results demonstrated that the DCs generated from all three techniques displayed a characteristic phenotype, with expression of MHC class I (HLA-ABC), MHC class II (HLA-DR), CD80, CD83 and CD86 (P>0.05; Fig. 1B). The survival of DCs following viral infection, was measured using trypan blue staining. The results showed that rAAV/AFP-pulsing or wild type AAV-pulsing has no significant effect on the viability of DCs (data not shown).

Proliferation of $T$ cells stimulated by rAAV/AFP-pulsed $D C s$. In order to assess the stimulatory capacity of DCs, rAAV/AFP-pulsed, lysate-pulsed, wild type AAV-pulsed and untreated DCs were co-cultured for eight days with T cells from the donors from which the DCs had been obtained. The formation of large $\mathrm{T}$ cell clusters was observed in $\mathrm{T}$ cells co-cultured with rAAV/AFP-pulsed DCs. Smaller clusters were observed in $\mathrm{T}$ cells that had been co-cultured with lysate-pulsed and untreated DCs (Fig. 2A). T cell proliferation was induced by rAAV/AFP-pulsed, lysate-pulsed, wild type AAV-pulsed and untreated DCs. However, proliferation levels were lower for the lysate-pulsed, wild type AAV-pulsed and untreated groups that for the rAAV/AFP-pulsed groups $(\mathrm{P}<0.05$; Fig. 2B and $\mathrm{C})$.

Cytokine production of $C D 4+$ and $C D 8+T$ cells. The cytokine production of $\mathrm{T}$ cells was measured using intracellular staining in order to determine $\mathrm{T}$ cell activation. $\mathrm{T}$ cells co-cultured with DCs were collected on day eight and analyzed for expression of IFN- $\gamma$ and IL-4. A relatively high level of IFN- $\gamma$ production was detected in CD4+ (30.2\%) and CD $8+(32.8 \%) \mathrm{T}$ cells stimulated by rAAV/AFP-pulsed DCs. A smaller proportion of IFN- $\gamma$ producing $\mathrm{T}$ cells were observed in the $\mathrm{T}$ cell populations co-cultured with either cancer cell lysate-pulsed DCs (10.5\% of CD4-positive cells and 9.6\% of CD8-positive cells) or untreated DCs (5.6 and $3.2 \%$ of CD4 and CD8-positive cells, respectively; $\mathrm{P}<0.05$ ). Minimal production of IL-4 was detected in CD4+ and CD8+ T cells (Fig. 3).

rAAV/AFP-pulsed DCs primed and propagated AFP-specific and HLA class I-restricted CTLs. T cells were co-cultured 

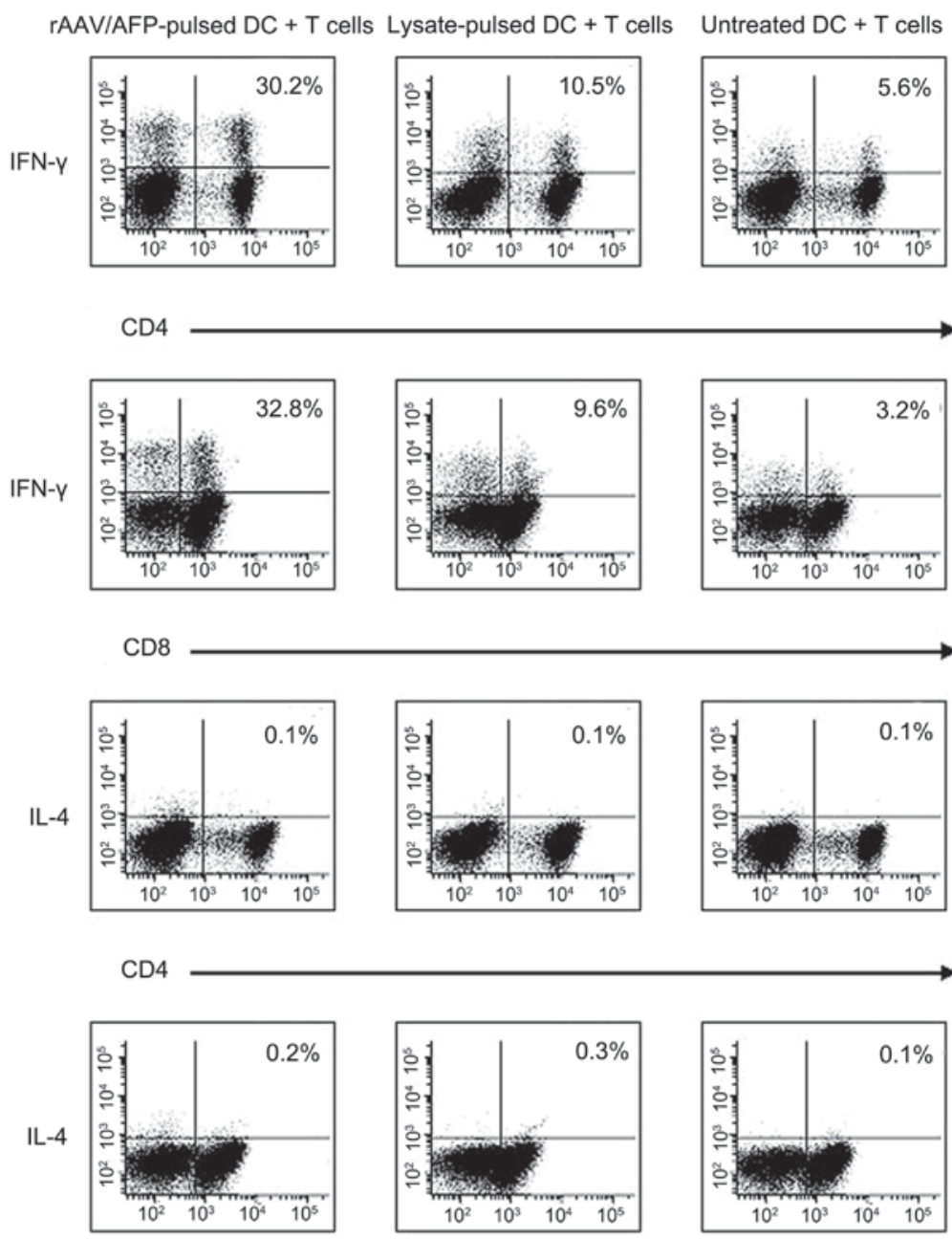

CD8

Figure 3. IFN- $\gamma$ and IL-4 secretion in $\mathrm{CD} 4^{+}$and $\mathrm{CD} 8^{+} \mathrm{T}$ cells that were stimulated by DCs, which had been pulsed by different techniques. IFN- $\gamma$, interferon- $\gamma$; IL-4, interleukin-4; DCs, dendritic cells; AFP, $\alpha$-fetoprotein; rAAV, recombinant adeno-associated virus.

with rAAV/AFP-pulsed, lysate-pulsed or untreated DCs at a ratio of 20:1 for eight days. A $6 \mathrm{~h}$ chromium-51 (51Cr) release assay was used to assess the induction of the CTL response. In order to determine antigen-specific and HLA class I-restricted tumor lysis by CTLs, multiple tumor targets were used (Fig. 4A). The T cells stimulated by rAAV/AFP-pulsed DCs exhibited $42.9 \%$ lysis of HepG2 (HLA-A2+, AFP+) cells and $48.5 \%$ lysis of BEL7402 (HLA-A2+, AFP+) cells. By contrast, there was reduced CTL activity against SMMC-7721 (HLA-A2+, AFP-), SK-Hep1 (HLA-A2+, AFP-) and K562 cells (HLA-A2-, AFP-; P<0.05). Furthermore, lysis of HepG2 and BEL7402 cells was significantly reduced by preincubation of the tumor cells with anti-HLA-A2 mAb (Fig. 4B). As shown in Fig. 4C, only $22.4 \%$ lysis of $\mathrm{HepG} 2$ cells was observed when using T cells stimulated by HepG2-lysate-pulsed DCs, which was significantly lower than that induced by $\mathrm{T}$ cells stimulated by rAAV/AFP-pulsed DCs $(\mathrm{P}<0.05)$. The killing activity of CTLs stimulated by lysate-pulsed DCs against BEL7402, SMMC-7721 and SK-Hep1 cells was observed. Cell death was not observed using the untreated DC-stimulated T cells (Fig. 4D).

\section{Discussion}

DCs are potent antigen-presenting cells that are involved in regulating immune responses (28-30). In human tumors, the presence of functional immunogenic DCs is rare. Defective DC recruitment, differentiation, maturation and survival may contribute to the low levels of functional DCs, observed in human cancer (31). An approach which may be effective in combatting these low levels is to generate functional DCs in vitro and transplant the genetically manipulated DCs into patients. Immunization of patients with ex vivo-generated DCs is feasible and enhances antigen-specific immune responses in humans.

In the present study,DCstransduced with antigen-expressing rAAV were compared with control lysate-pulsed DCs. The results demonstrated that direct transduction of DCs with antigen-expressing rAAV was more efficient than loading DCs with tumor lysate. There are a number of possible reasons that may explain this result. Due to protein degradation and MHC molecule cycling, protein pulsing of DCs may be an inefficient way to deliver an antigen to target cells. By contrast, gene transfer ensures long-lasting expression of the target antigen 
A
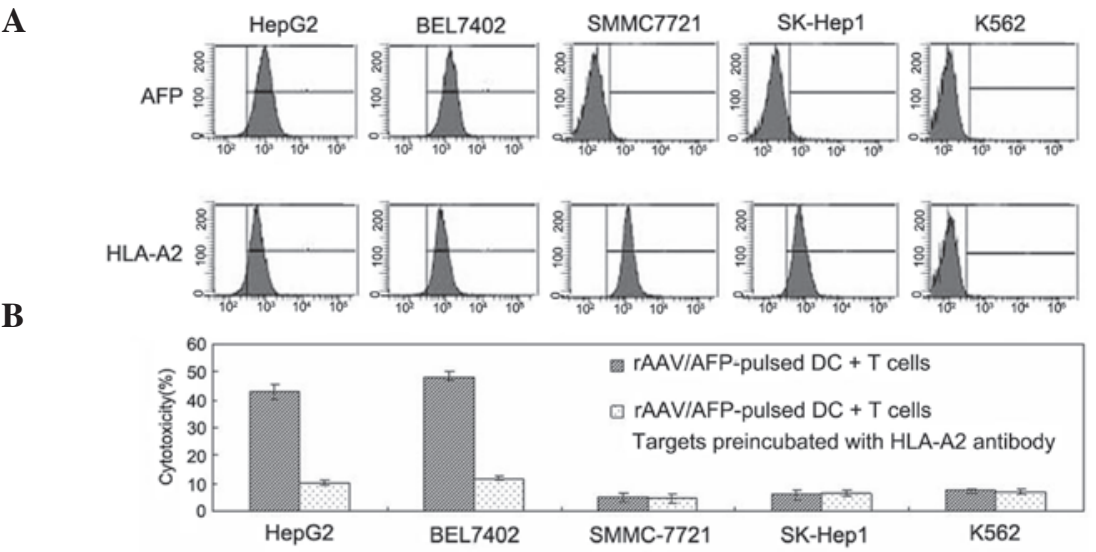

C

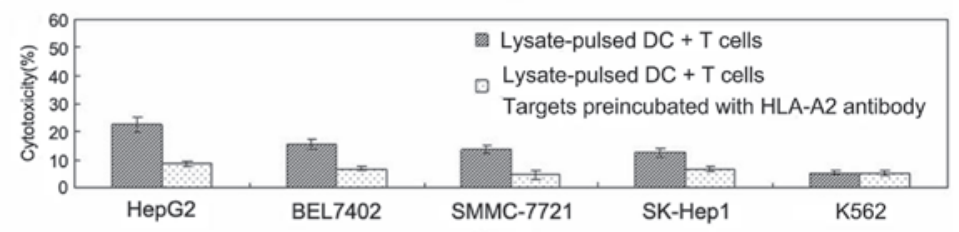

D

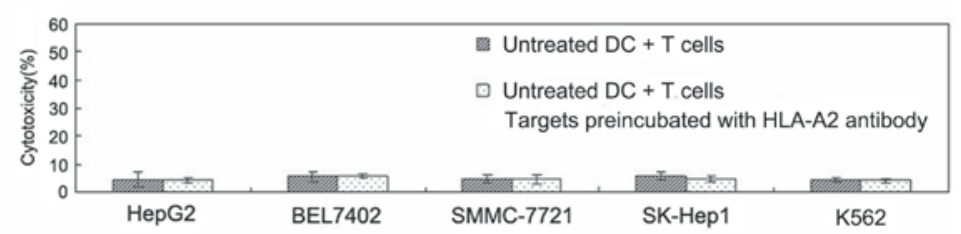

Figure 4. Stimulation of antigen-specific CTLs by DCs pulsed by different techniques. (A) AFP and HLA-A2 expression in target cells. (B, C and D) CTL activity of T cells stimulated by DCs pulsed by different techniques against indicated targets. CTLs, cytotoxic lymphocytes; DCs, dendritic cells; AFP, $\alpha$-fetoprotein; HLA-A2, human leukocyte antigen-A2; rAAV, recombinant adeno-associated virus.

and may provide an opportunity for the repeated stimulation of CTLs. Viral entry into DCs is often more efficient than other approaches for the introduction of antigen. Viral delivery of antigens may result in the production of an entire array of epitopes presented by the autologous MHC class I and MHC class II molecules, which results in a more efficient activation of multiclonal T cells (32-34). Viral gene delivery results in the production and endogenous expression of a number of epitopes in DCs, which are theoretically more representative and immunoreactive than that produced by alternative approaches.

AFP is an ideal antigen target for immunotherapy in HCC, due to the high level of expression in the majority of HCCs, and the low level of expression in healthy liver and other cell types. The present study demonstrated that the RAAV/AFP virus effectively loaded freshly adherent DC precursors with the AFP gene. In addition, the transduction of the AFP gene into DCs resulted in functionally active AFP-epitope-presenting DCs, without impacting upon the phenotype, viability and functions of these cells. One explanation for this result may be that rAAV is one of the safest virus vectors used in gene therapy. The wild-type virus has never been shown to cause human disease.

The rAAV/AFP-pulsed DCs were able to prime and propagate AFP antigen-specific CTLs in an efficient manner. A notable phenomenon observed during the priming process was the formation of large DC-T cell clusters two days after addition of $\mathrm{T}$ cells, which may suggest enhanced priming in this group (35-37). T cell proliferation were also induced by wild type AAV-pulsed DCs. However, the proliferation level was significantly lower in this group, which indicates that proliferation is specific for AFP, but not for the cytokines produced by DCs in response to a viral infection.

The T cells primed by rAAV/AFP-pulsed DCs exhibited low levels of IL-4, a characteristic cytokine in the Th2 response, and increased levels of IFN- $\gamma$, a typical Th1 cytokine. These data suggest that the use of rAAV/AFP-pulsed DCs is effective in generating a significant Th1 response.

Marked CTL activity, induced by rAAV/AFP-pulsed DCs against MHC class I-matched, AFP-positive HCC cell lines was observed after eight days of priming. The majority of DC loading/priming protocols require a far longer period in order to demonstrate CTL activity. The underlying reason for this is unknown. However, there are a number of possible explanations. Firstly, the high transduction efficiency of the rAAV vector. Secondly, the effective formation of the DC-T cell clusters and proliferation of T cells. Thirdly, the high levels of CD80, CD83 and CD86 expression. Finally, higher IFN- $\gamma$ secretion with no detectable IL-4 secretion, which is associated with the desirable Th1 response. Blockage of HLA-A2 inhibited cytotoxity, which was in accordance with HLA-A2-restriction. No significant cytotoxicity was observed against AFP-negative target cells or NK-sensitive K562 cells, indicating that the cytotoxicity is antigen-specific and MHC class I restricted.

In conclusion, rAAV/AFP pulsing of DCs represents a promising technique for effectively introducing the AFP antigen gene into DCs in order to stimulate an AFP-specific CTL response against HCC. The protocol described in the current study may 
in future be used to develop an adjuvant immunotherapy for the treatment of HCC. In addition, the present study provides a foundation for future studies, involving transduction of alternative tumor antigen genes into DCs using rAAV in order to elicit a CTL response against the transduced antigen.

\section{References}

1. Liu Y, Daley S, Evdokimova VN, Zdobinski DD, Potter DM and Butterfield LH: Hierarchy of alpha fetoprotein (AFP)-specific $\mathrm{T}$ cell responses in subjects with AFP-positive hepatocellular cancer. J Immunol 177: 712-721, 2006.

2. Butterfield LH, Koh A, Meng W, et al: Generation of human $\mathrm{T}$ cell responses to an HLA-A2.1-restricted peptide epitope from alpha-fetoprotein. Cancer Res 59: 3134-3142, 1999.

3. Butterfield LH, Meng WS, Koh A, et al: T cell responses To HLA-A*0201-restricted peptides derived from human alpha fetoprotein. J Immunol 166: 5300-5308, 2001.

4. Steinman RM: The dendritic cell system and its role in immunogenicity. Ann Rev Immunol 9: 271-296, 1991.

5. Zitvogel L, Mayordomo JI, Tjandrawan T, DeLeo AB, Clarke MR, Lotze MT and Storkus WJ: Therapy of murine tumors with tumor peptide-pulsed dendritic cells: dependence on T cells, B7 costimulation and T helper cell I-associated cytokines. J Exp Med 183: 183-187, 1996.

6. Philip R, Brunette E, Ashton J, et al: Tansgene expression in dendritic cells to induce antigen-specific cytotoxic $\mathrm{T}$ cells in healthy donors. Cancer Gene Ther 5: 236-246, 1998.

7. McArthur JG and Mulligan RC: Induction of protective anti-tumor immunity by gene-modified dendritic cells. J Immunother 21 : 41-47, 1998.

8. Palmer DH, Midgley RS, Mirza N, et al: A phase II study of adoptive immunotherapy using dendritic cells pulsed with tumor lysate in patients with hepatocellular carcinoma. Hepatology 49: 124-132, 2009.

9. Romani N, Gruner S, Brang D, et al: Proliferating dendritic cell progenitors in human blood. J Exp Med 180: 83-93, 1994.

10. Sallusto F and Lanzavecchia A: Efficient presentation of soluble antigen by cultured human dendritic cells is maintained by granulocyte/macrophage colony stimulating factor plus interleukin 4 and down regulated by tumor necrosis factor alpha. J Exp Med 179: 1109-1118, 1994.

11. Young JW and Inaba K: Dendritic cells as adjuvants for class I major histocompatibility complex-restricted antitumor immunity. J Exp Med 183: 7-11, 1996.

12. Paglia P, Chiodoni C, Rodolfo M and Colombo MP: Murine dendritic cells loaded in vitro with soluable protein prime cytotoxic T lymphocytes against tumor antigen in vivo. J Exp Med 183: 317-322, 1996.

13. Alexander M, Salgaller ML, Celis E, Sette A, Barnes WA, Rosenberg SA and Steller MA: Generation of tumor-specific cytotoxic T lymphocytes from peripheral blood of cervical cancer patients by in vitro stimulation with a synthetic human papillomavirus type 16 E7 epitope. Am J Obstet Gynecol 175: 1586-1593, 1996

14. Sonderbye L, Feng S, Yacoubian S, Buehler H, Ahsan N, Mulligan $\mathrm{R}$ and Langhoff $\mathrm{E}$ : In vivo and in vitro modulation of immune stimulatory capacity of primary dendritic cells by adenovirus-mediated gene transduction. Exp Clin Immunogenet 15: 100-111, 1998.

15. Kim CJ, Prevette T, Cormier J, et al: Dendritic cells infected with poxviruses encoding MART-1/Melan A sensitize T lymphocyte in vitro. J Immunother 20: 276-286, 1997.

16. Liu Y, Butterfield LH, Fu X, et al: Lentivirally Engineered DC activate AFP-specific T cells which Inhibit Hepatocellular Carcinoma Growth in vitro and in vivo. Int J Oncol 39: 245-253, 2011.
17. Jooss K, Yang Y, Fisher KJ and Wilson JM: Transduction of dendritic cells by DNA viral vectors directs the immune response to transgene products in muscle fibers. J Virol 72: 4212-4223, 1998.

18. Yang Y, Su Q and Wilson JM: Role of viral antigens in destructive cellular immune responses to adenovirus vector-transduced cells in mouse lungs. J Virol 70: 7209-7212, 1996.

19. Veron P, Allo V, Rivière C, Bernard J, Douar AM and Masurier C: Major subsets of human dendritic cells are efficiently transduced by self-complementary adeno-associated virus vectors 1 and 2 . J Virol 81: 5385-5394, 2007.

20. Dullaers M and Thielemans K: From pathogen to medicine: HIV-1-derived lentiviral vectors as vehicles for dendritic cell based cancer immunotherapy. J Gene Med 8: 3-17, 2006.

21. Warrington KH Jr and Herzog RW: Treatment of human disease by adeno-associated viral gene transfer. Hum Genet 119: 571-603, 2006.

22. Chiriva-Internati M, Liu Y, Salati E, et al: Efficient generation of cytotoxic $\mathrm{T}$ lymphocytes against cervical cancer cells by adeno-associated virus/human papillomavirus type $16 \mathrm{E} 7$ antigen gene transduction into dendritic cells. Eur J Immunol 32: 30-38, 2002.

23. Samulski RJ, Berns KI, Tan M and Muzyczka N: Cloning of adeno-associate d virus into pBR322: rescue of intact virus from the recombinant plasmid in human cells. Proc Natl Acad Sci 79: 2077-2081, 1982.

24. Morinaga T, Sakai M, Wegmann TG and Tamaoki T: Primary structures of human alpha-fetoprotein and its mRNA. Proc Natl Acad Sci 80: 4604-4608, 1983.

25. Collaco RF, Cao X and Trempe JP: A helper virus-free packaging system for recombinant adeno-associated virus vectors. Gene 238: 397-405, 1999.

26. Auricchio A, Hildinger M, O'Connor E, Gao GP and Wilson JM: Isolation of highly infectious and pure adeno-associated virus type 2 vectors with a single-step gravity-flow column. Hum Gene Ther 12: 71-76, 2001

27. Devos R, Cheroutre H, Taya Y, Degrave W, Van Heuverswyn H and Fiers W: Molecular cloning of human immune interferon cDNA and its expression in eukaryotic cells. Nucleic Acids Res 10: 2487-2501, 1982.

28. Steinman RM: Dendritic cells: from the fabric of immunology. Clin Invest Med 27: 231-236, 2004.

29. Bender A, Sapp M,Feldman M, et al: Dendritic cells as immunogens for human CTL responses. Adv Exp Med Biol 417: 383-387, 1997.

30. Sheng KC, Pietersz GA, Wright MD and Apostolopoulos V: Dendritic cells: activation and maturation-applications for cancer immunotherapy. Curr Med Chem 12: 1783-1800, 2005.

31. Zou W: Immunosuppressive networks in the tumor environment and their therapeutic relevance. Nat Rev Cancer 5: 263-274, 2005.

32. Nonacs R1, Humborg C, Tam JP and Steinman RM: Mechanisms of mouse spleen dendritic cell function in the generation of influenza-specific cytolytic T lymphocytes. J Exp Med 176: 519-529, 1992.

33. Banchereau J, Briere F, Caux C, et al: Immunobiology of dendritic cells. Annu Rev Immunol 18: 767-811, 2000.

34. Jaraquemada D, Marti $M$ and Long EO: An endogenous processing pathway in vaccinia virus-infected cells for presentation of cytoplasmic antigens to class II-restricted T cells. J Exp Med 172: 947-954, 1990.

35. Schuhbauer DM, Mitchison NA and Mueller B: Interaction within clusters of dendritic cells and helper T cells during initial Th1/Th2 commitment. Eur J Immunol 30: 1255-1262, 2000.

36. McClellan AD, Heiser A and Hart DN: Induction of dendritic cell costimulator molecule expression is suppressed by $\mathrm{T}$ cells in the absence of antigen-specific signalling: role of cluster formation, CD40 and HLA-class II for dendritic cell activation. Immunol 98: 171-180, 1999.

37. Stuhler G, Zobywalski A, Grünebach F, et al: Immune regulatory loops determine productive interactions within human T lymphocyte-dendritic cell clusters. Proc Natl Acad Sci USA 96: 1532-1535, 1999. 